\title{
Efeito do método Pilates sobre a escoliose idiopática: estudo de caso
}

\author{
Effect of the Pilates method on idiopathic scoliosis: a case study \\ Pâmela Miotti de Moura', Marília Luz da Silva1 ${ }^{1}$, Lilian Pinto Teixeira², Eloá Ferreira Yamada ${ }^{3}$, Simone Lara ${ }^{4}$ \\ ${ }^{1}$ Acadêmicas do Curso de Fisioterapia da Universidade Federal do Pampa (UNIPAMPA). \\ ${ }^{2}$ Fisioterapeuta do Curso de Fisioterapia da UNIPAMPA. \\ ${ }^{3}$ Fisioterapeuta, Mestre em Engenharia Biomédica, Docente do Curso de Fisioterapia da UNIPAMPA. \\ ${ }^{4}$ Fisioterapeuta, Doutora em Ciências pela Universidade Federal de Santa Maria (UFSM), Docente do Curso de Fisioterapia da UNIPAMPA.
}

\section{RESUMO}

Objetivos: Avaliar os efeitos do método Pilates, em curto prazo, nas alterações posturais, na flexibilidade e na força dos músculos flexores e extensores de tronco em uma paciente portadora de escoliose idiopática.

Descrição do Caso: Trata-se de um estudo prospectivo no qual foram avaliadas as seguintes alterações em uma menina de 11 anos de idade, com escoliose idiopática, antes após um protocolo de tratamento pelo método Pilates:1) alterações posturais em vista anterior, posterior e de perfil, analisadas por meio do Software de Avaliação Postural SAPO; 2) flexibilidade de cadeia posterior, avaliada pelo teste de sentar e alcançar e distância dedo-chão; e 3) força muscular de flexores e extensores de tronco, avaliada pelo teste manual de força muscular. O protocolo de tratamento incluiu exercícios do método Pilates solo e acessórios, constando de 10 sessões, três vezes por semana, durante um mês. Após a intervenção, a paciente apresentou melhora postural no alinhamento de cabeça, ombros, escápulas, ângulo de Talles, tronco, abdômen e pelve. A flexibilidade e a força dos músculos flexores e extensores de tronco apresentaram melhora quando comparadas entre antes e após as sessões fisioterapêuticas.

Conclusões: O caso estudado sugere efeitos positivos do método Pilates, em curto prazo, em adolescente com diagnóstico clínico de escoliose idiopática.

DESCRITORES: ESCOLIOSE; ADOLESCENTE; FISIOTERAPIA; POSTURA; REABILITAÇÃO.

\section{ABSTRACT}

Aims: To investigate the short-term effects of the Pilates method on postural alterations, flexibility and strength of the flexor and extensor muscles of the trunk in a patient with idiopathic scoliosis.

Case Description: This is a prospective study in which the following alterations in an 11-year-old girl with idiopathic scoliosis were evaluated before and after a treatment protocol based on the Pilates method: 1) postural alterations in anterior, posterior and profile view, measured using the Postural Assessment Software (Software de Avaliação Postural, SAPO, in Portuguese); 2) posterior chain flexibility, assessed using the sit-and-reachand the finger-floor distance tests; and 3) strength of the flexor and extensor muscles of the trunk, assessed using manual muscle strength testing. The treatment protocol included Pilates mat and equipment exercises, and consisted of 10 sessions given three times a week for a month. After the intervention, the patient showed improvement in postural alignment of the head, shoulders, scapulae, Thales' angle, trunk, abdomen and pelvis. We identified improvements in flexibility and strength of the flexor and extensor muscles of the trunk, when comparing values before and after the physical therapy sessions.

Conclusions: This case study suggests positive, short-term effects of Pilates exercise on an adolescent with a clinical diagnosis of idiopathic scoliosis.

KEY WORDS: SCOLIOSIS; ADOLESCENT; PHYSICAL THERAPY; POSTURE; REHABILITATION. 


\section{INTRODUÇÃO}

A escoliose idiopática consiste em uma alteração tridimensional da coluna vertebral, de etiologia desconhecida. Frequentemente inicia-se na puberdade, tendo grande momento de progressão associado ao estirão de crescimento. Trata-se de condição altamente prevalente e de bom prognóstico na maioria dos casos. Considerando que a estrutura óssea tem seu crescimento e desenvolvimento completo em torno da segunda década de vida, é importante ressaltar a importância de intervenções conservadoras, para evitar a progressão da curva escoliótica, de forma precoce, enquanto não atingir a maturação da estrutura óssea. ${ }^{1-5}$

Segundo Yaman e Dalbayrak, ${ }^{6}$ uma das alternativas para o tratamento conservador da escoliose é a intervenção fisioterapêutica, baseada em um programa de exercícios específicos para essa condição. Alguns dos métodos utilizados são os exercícios de estabilização lombo-pélvica, ${ }^{7}$ reeducação postural global, ${ }^{8}$ Iso Stretching 9 e Pilates. ${ }^{10}$

$\mathrm{O}$ método Pilates caracteriza-se por movimentos projetados de forma que os praticantes mantenham a posição neutra da coluna vertebral, minimizando o recrutamento muscular desnecessário, prevenindo a fadiga precoce e a diminuição da estabilidade corporal. A prática desse método acarreta benefícios sobre a flexibilidade global, o alinhamento postural e a coordenação motora, além do aumento da força muscular, o que demonstra uma relação direta com o processo de reeducação postural. ${ }^{10-11}$

Contudo, existem poucos dados reportando os efeitos desse método sobre a escoliose de adolescentes num curto período de tempo. Assim, o presente estudo teve por finalidade avaliar o caso de uma paciente portadora de escoliose idiopática lombar à direita, na qual foram investigados os efeitos do método Pilates, aplicado em curto prazo, nas alterações posturais, na flexibilidade e na força muscular de flexores e de extensores de tronco.

\section{RELATO DO CASO}

\section{Descrição do caso}

Este trabalho consta de um estudo prospectivo de uma adolescente de 11 anos de idade, branca, estudante do sexto ano do ensino fundamental, com diagnóstico clínico de escoliose idiopática. $\mathrm{O}$ estudo avaliou a atuação fisioterapêutica, baseada nos exercícios do método Pilates, como forma de reabilitação dessa disfunção.
A paciente não apresentava histórico de traumas musculoesqueléticos ou cirurgias prévias e nunca havia realizado tratamento fisioterapêutico. Ela relatou ter começado a sentir dor leve na região cervical e nos ombros um ano antes. Afirmava ficar por longos períodos na posição sentada, na escola e em casa (em frente ao computador e à televisão), sendo que nessa posição referia dor também na região da coluna torácica baixa. Referia sentir dificuldade em manter a postura ereta, especialmente para sentar corretamente. $\mathrm{Na}$ radiografia foi possível constatar, através do laudo médico, que a escoliose era de característica idiopática não estrutural lombar à direita, porém o laudo não mencionava o ângulo de Cobb.

Os atendimentos fisioterapêuticos foram realizados ao longo de quatro semanas, constando de três sessões semanais, com duração de uma hora cada, totalizando 10 sessões, além de uma sessão inicial de avaliação e uma final de reavaliação. $\mathrm{O}$ estudo foi realizado no setor de Fisioterapia do Posto de Estratégia de Saúde da Família II, em Uruguaiana, Rio Grande do Sul.

O estudo foi conduzido dentro dos padrões exigidos pela Declaração de Helsinque e aprovado pelo Comitê de Ética e Pesquisa da Universidade Federal do Pampa (UNIPAMPA), em consonância com a resolução 466/12, do Conselho Nacional de Saúde, sob parecer n. 457.088, de novembro de 2013. Os princípios éticos foram respeitados, e a responsável pela participante assinou um termo de consentimento livre e esclarecido.

\section{Protocolos de avaliação}

Todos os testes relativos à avaliação foram realizados antes e após a intervenção fisioterapêutica pelo mesmo pesquisador, consistindo nos protocolos descritos a seguir.

- Avaliação postural: foram analisados os itens posicionamento da cabeça, da cintura escapular, dos membros superiores, da coluna cervical, torácica e lombar, da pelve, do abdômen e dos membros inferiores. A paciente foi previamente observada por meio da simetrografia, com o posicionamento ortostático com vistas anterior, posterior e perfil direito e esquerdo. Os dados foram registrados em uma ficha de avaliação e por meio de fotografias que foram analisadas no Software para Avaliação Postural SAPO. Os pontos anatômicos utilizados na avaliação com Software SAPO partiram de um protocolo próprio para abranger pontos específicos, fundamentais à pesquisa. Os pontos analisados e demarcados foram: trago direito e esquerdo, acrômio direito e esquerdo, espinha ilíaca ântero-superior direita e esquerda, 
trocânter maior do fêmur direito e esquerdo, linha articular do joelho direita e esquerda, ponto central da patela direita e esquerda, tuberosidade da tíbia direita e esquerda, maléolo lateral direito e esquerdo, processos espinhosos de C7 e T3, ângulo inferior da escápula direita e esquerda, ponto sobre a linha média da perna direita e esquerda, ponto entre a segunda e terceira cabeça do metatarso direita e esquerda, ponto sobre o tendão calcâneo na altura média dos dois maléolos direito e esquerdo, calcâneo direito e esquerdo..$^{11,12}$

- Teste de sentar e alcançar com o auxílio do banco de Wells: para a realização do teste, a participante permaneceu sentada com os joelhos estendidos, membros inferiores levemente separados, pés apoiados firmemente na parede da caixa de madeira (banco de Wells), ombros fletidos e cotovelos estendidos. A partir dessa posição, a participante realizou um movimento à frente com o tronco, tentando alcançar com as mãos sobrepostas o maior deslocamento possível conseguido, mantendo essa posição momentaneamente sobre uma escala graduada em centímetros na parte superior da caixa, sendo registrada a média das três tentativas em centímetros. ${ }^{14}$ De acordo com Projeto Esporte Brasil (PROESP) do Ministério do Esporte, ${ }^{15}$ no teste de sentar e alcançar com auxílio do banco de Wells, para o gênero e a idade (feminino, 11 anos), os valores de normalidade estão entre 22 e $28 \mathrm{~cm}$.

- Teste de distância dedo-chão: a paciente iniciou o teste em posição ortostática e, a seguir, foi solicitado que realizasse uma flexão anterior máxima de tronco, deslizando suas mãos à frente do corpo, mantendo os joelhos estendidos durante todo o processo. Ao final, a distância de seu terceiro dedo até o chão foi medida com uma fita métrica. A medida da amplitude de movimento (ADM) de flexão anterior do tronco é considerada como sendo a medida da distância total (medida dedo-chão). Quanto menor a medida dedo-chão obtida, maior será a ADM do indivíduo. ${ }^{16}$

- Teste manual de força muscular de flexores e extensores de tronco: realizado em decúbito dorsal e ventral, respectivamente, no qual foi solicitado à paciente realizar a flexão e extensão do tronco com resistência do terapeuta. A avaliação clínica da força muscular baseia-se na escala de seis níveis, proposta por Daniels, Williams e Worthingham, ${ }^{17}$ sendo: $0=$ sem evidência de contração muscular; $1=$ evidência de contração muscular, sem movimento articular; $2=$ amplitude de movimento incompleta; 3 =amplitude de movimento completa contra a gravidade; 4=amplitude de movimento completa contra a gravidade e resistência manual sub-máxima; e 5=amplitude de movimento completa contra a gravidade e resistência manual máxima.

\section{Protocolo de intervenção}

O protocolo de intervenção baseou-se nos exercícios do método Pilates (Pilates solo e acessórios),dividido em três etapas, conforme Araújo et al., ${ }^{18} \mathrm{e}$ adaptado pelos autores, constando de preparação, parte específica e volta à calma.

\section{Preparação}

- Rolamento para baixo em pé: paciente em pé, pés paralelos afastados à largura do quadril com a coluna neutra, inspirar e expirar ao iniciar a flexão do tronco a partir da cabeça em direção aos pés, até atingir a flexão completa, inspirando em baixo e expirando para subir a partir do cóccix, mobilizando as vértebras até retornar a posição inicial.

- Alongamento em decúbito dorsal: paciente na bola suíça, com extensão dos joelhos e membros superiores flexionados acima da cabeça, mantendo a posição por 10 respirações.

- Alongamento em decúbito ventral: paciente na bola suíça, mantendo os membros superiores e inferiores relaxados durante 10 respirações.

\section{Parte específica}

Nessa etapa foram utilizados exercícios específicos do método Pilates solo e com acessórios (bola suíça e meia bola ou bosu), sendo realizada uma série de 10 repetições para cada exercício (Figura 1).

- Cisne: paciente em decúbito ventral na bola suíça, com as mãos na coluna cervical, inspira em repouso, expira realizando a extensão da coluna torácica e inspira novamente para retornar à posição inicial (Figura 1a e b).

- Elevação torácica: paciente em decúbito dorsal no colchonete, com flexão de joelhos e quadris, pés apoiados no chão e mãos atrás da cabeça, solicitase que ao expirar realize flexão da coluna torácica, mantendo a pelve em posição neutra e inspirando para voltar à posição inicial (Figura 1c e d).

- Sereia: paciente sentada ao lado do bosu com os joelhos flexionados para o lado contralateral, inspirar em repouso e expirar fazendo flexão lateral da coluna para o lado das pernas, sempre mantendo o contato dos ísquios com o colchonete, mantém a posição na inspiração e, expirando, retorna à posição inicial (Figura 1 e e f).

- Serrote: Paciente sentada no bosu com joelhos estendidos, coluna neutra e membros superiores 
abduzidos no plano da escápula, expira ao realizar rotação com inclinação lateral de coluna, levando o braço em direção ao tornozelo da perna oposta, mantém o alongamento durante a inspiração e, expirando, retorna à posição inicial (Figura $1 \mathbf{g} \mathrm{e} \mathbf{h}$ ).

\section{Volta à calma}

Esse procedimento foi composto de três movimentos, tendo duração de cinco minutos, sendo realizadas três repetições para cada movimento. Os movimentos foram executados visando o relaxamento e o alongamento da musculatura trabalhada:
- participante em decúbito lateral sobre a bola do lado da convexidade da curva escoliótica;

- em seguida, deitada em decúbito ventral na bola, mantém o apoio das pontas dos pés e das mãos;

- por último, deitada em decúbito dorsal na bola, mantém os pés apoiados e os membros superiores para trás.

Este estudo de caso caracteriza-se por ser do tipo $\mathrm{AB}$ experimental $(\mathrm{A}=$ avaliação; $\mathrm{B}=$ reavaliação $)$. $\mathrm{As}$ variáveis de flexibilidade e força foram analisadas por estatística descritiva a partir dos dados coletados antes e depois das sessões de Pilates.

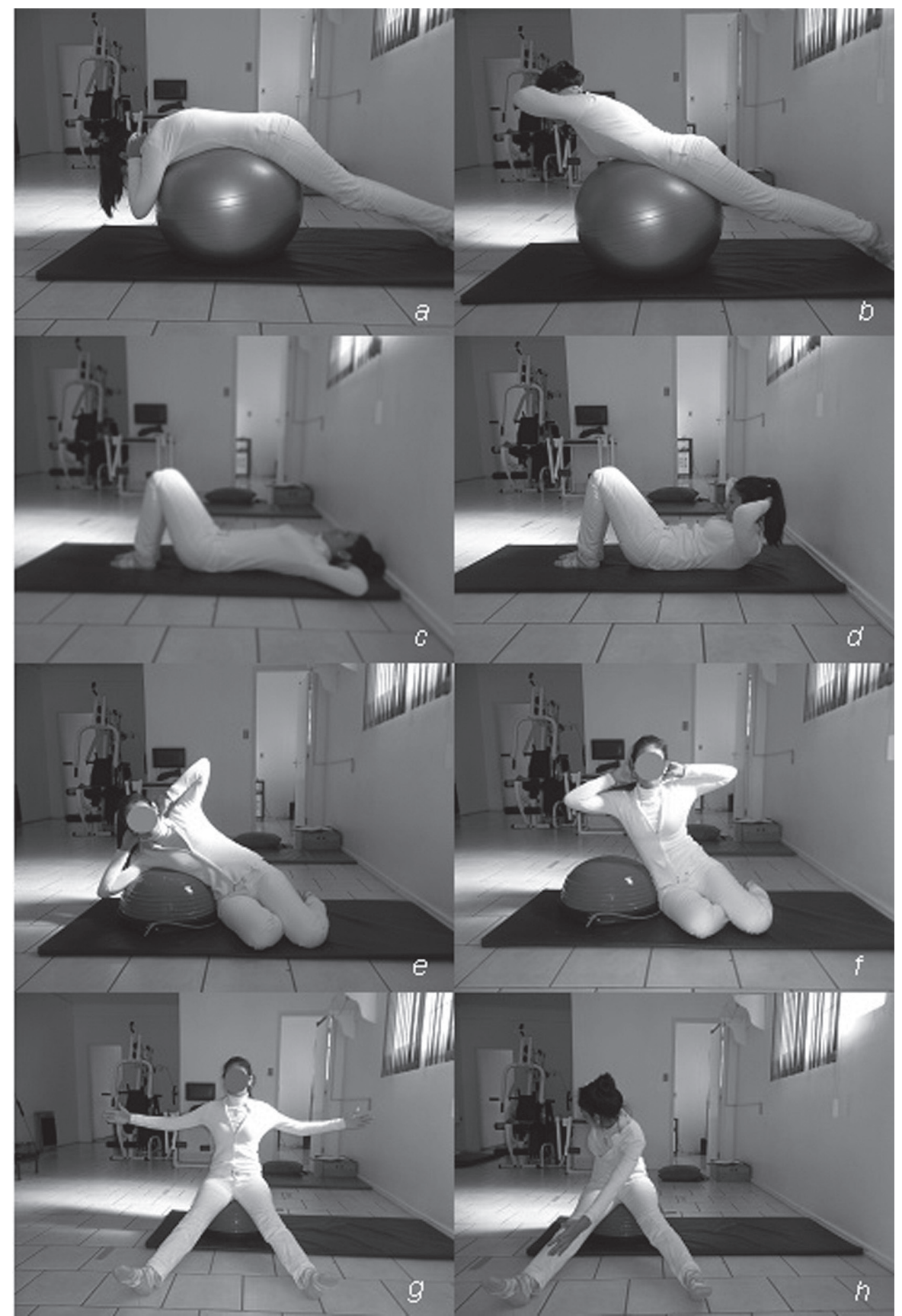

Figura 1. Exercícios específicos do método Pilates:

a - posição inicial do exercício cisne;

b - posição final do exercício cisne;

c - posição inicial da elevação torácica;

d - posição final da elevação torácica;

e - posição inicial da sereia:

f - posição final da sereia;

g - posição inicial do serrote;

h - posição final do serrote.

Fonte: os autores, 2014. 


\section{Avaliações}

As alterações posturais encontradas por meio do software SAPO que se apresentaram mais evidentes na avaliação inicial encontram-se descritas na Tabela 1.

Após a realização do protocolo do método Pilates, a participante apresentou evolução mais expressiva em relação à simetria da cabeça, dos ombros, das escápulas, dos ângulos de Talles e das espinhas ilíacas ânterosuperiores. Ainda, houve melhora na protrusão da cabeça, na anteversão pélvica, na protrusão abdominal, e no alinhamento axial do tronco (Tabela 2).

A melhora da flexibilidade muscular foi observada por meio do teste de sentar e alcançar com auxílio do banco de Wells e da distância dedo-chão, sendo as diferenças pré e pós-intervenção de $4,5 \mathrm{~cm}$ e $4,0 \mathrm{~cm}$ respectivamente (Tabela $\mathbf{3}$ ).

Em relação à força muscular de flexores e extensores de tronco, houve uma melhora de grau 4 (bom) para 5 (normal), quando comparada antes e após as sessões fisioterapêuticas (Tabela 3).

\section{DISCUSSÃO}

A literatura descreve a escoliose idiopática do adolescente como uma disfunção frequente, mais comum no gênero feminino e ocorrendo especialmente dos nove aos 13 anos de idade, com progressão associada ao estirão de crescimento. O momento de maior risco para a progressão da escoliose idiopática é o início da puberdade, período no qual o crescimento ósseo ocorre de forma muito rápida, principalmente em função das alterações hormonais. ${ }^{19-21}$ Este perfil vai ao encontro do caso avaliado no presente estudo, de uma adolescente de 11 anos de idade, faixa etária que condiz com o início da puberdade, quando se iniciaram os primeiros sintomas de desconforto muscular.

Além das influências hormonais, fatores de ordem ambiental e comportamental podem atuar na escoliose do adolescente. O peso excessivo e o transporte inadequado do material escolar, as más condições ergonômicas de mobiliários inadequado e posturas incorretas durante as aulas e fora da escola podem contribuir para a evolução da escoliose nos escolares. ${ }^{22}$ A escoliose da participante do presente estudo foi descrita como sendo do tipo não estrutural, ou seja, possivelmente associada a maus hábitos posturais e de caráter reversível com o tratamento conservador.

No caso em estudo, logo que os sintomas iniciaram foi buscado o atendimento médico. Ao ser diagnosticada a escoliose não estrutural, a paciente recebeu encaminhamento para a fisioterapia, fato
Tabela 1. Alterações posturais encontradas na avaliação inicial da paciente, analisadas por meio do SAPO

\begin{tabular}{cl}
\multicolumn{1}{c}{ Avaliação } & \multicolumn{1}{c}{ Alterações posturais } \\
Vista anterior & $\begin{array}{l}\text { Ângulo formado pelo alinhamento entre dois } \\
\text { pontos em relação a horizontal: cabeça rodada à } \\
\text { direita (pontos 2-3), ombro direito mais elevado } \\
\text { (pontos 4-5), espinha ilíaca ântero-superior esquerda } \\
\text { mais elevada, ângulo de Talles maior à esquerda } \\
\text { (ângulo entre os pontos 2-3 e 4-5). }\end{array}$ \\
Vista posterior & $\begin{array}{l}\text { Assimetria de escápulas, sendo que a direita } \\
\text { apresentou rotação externa, abdução e elevação. }\end{array}$ \\
Vista lateral & $\begin{array}{l}\text { Ângulo formado pelo alinhamento entre dois pontos } \\
\text { em relação à horizontal: cabeça anteriorizada } \\
\text { (pontos 2-5), quadril flexionado (pontos 5-23-30), } \\
\text { anteversão da pelve (pontos 21-22), e alinhamento } \\
\text { vertical do tronco (5-23). }\end{array}$ \\
\hline
\end{tabular}

Tabela 2. Evolução das principais alterações posturais (em graus) antes (avaliação inicial) e após (reavaliação) a intervenção fisioterapêutica, analisadas por meio do software SAPO

\begin{tabular}{|c|c|c|}
\hline & $\begin{array}{l}\text { Avaliação } \\
\text { inicial }\end{array}$ & Reavaliação \\
\hline \multicolumn{3}{|l|}{ Vista Anterior } \\
\hline \multicolumn{3}{|l|}{ Cabeça: } \\
\hline \multicolumn{3}{|l|}{ Tronco: } \\
\hline $\begin{array}{l}\text { - alinhamento horizontal das espinhas } \\
\text { ilíacas antero-superiores }\end{array}$ & $2,8^{\circ}$ & $2,1^{\mathrm{o}}$ \\
\hline $\begin{array}{l}\text { - ângulo entre os dois acrômios e as } \\
\text { duas espinhas ilíacas ântero-superiores } \\
\text { (ângulo de Talles) }\end{array}$ & $3,5^{\circ}$ & $0,2^{\circ}$ \\
\hline \multicolumn{3}{|l|}{ Vista Posterior } \\
\hline $\begin{array}{l}\text { Tronco: } \\
\text { - assimetria horizontal da escápula em } \\
\text { relação à T3 }\end{array}$ & $28,6^{\circ}$ & $13,7^{\circ}$ \\
\hline \multicolumn{3}{|l|}{ Vista Lateral } \\
\hline $\begin{array}{l}\text { Cabeça: } \\
\text { - alinhamento vertical da cabeça } \\
\text { (acrômio) }\end{array}$ & $10,8^{\mathbf{o}}$ & $2,5^{\circ}$ \\
\hline \multicolumn{3}{|l|}{ Tronco: } \\
\hline - alinhamento vertical do tronco & $-0,2^{\mathrm{o}}$ & $-0,8^{\circ}$ \\
\hline - ângulo do quadril (tronco e coxa) & $-1,90$ & $-2,8^{\circ}$ \\
\hline - alinhamento horizontal da pelve & $-12,5^{0}$ & $-27^{\circ}$ \\
\hline
\end{tabular}

Tabela 3. Flexibilidade de cadeia posterior e força muscular de flexores e extensores de tronco, antes e depois da intervenção

\begin{tabular}{lcc}
\multicolumn{1}{c}{ Avaliação } & Antes & Depois \\
\hline Flexibilidade - teste de sentar alcançar $(\mathrm{cm})$ & 21,3 & 25,8 \\
Flexibilidade - teste distância dedo-chão $(\mathrm{cm})$ & 18 & 14 \\
Força muscular - flexores de tronco & 4 & 5 \\
Força muscular - extensores de tronco & 4 & 5 \\
\hline
\end{tabular}


este que aumenta as probabilidades de correção da curvatura escoliótica, por meio da intervenção precoce. A escoliose ainda flexível e não estruturada apresenta uma probabilidade de correção e/ou estabilização postural maior, enquanto que ao final do crescimento, sua probabilidade de correção é menor. ${ }^{23}$

A intervenção fisioterapêutica proposta no presente estudo indicou que o método Pilates, em curto prazo, trouxe melhoras à participante nos aspectos relacionados às alterações posturais, flexibilidade da cadeia muscular posterior e força dos músculos flexores e extensores do tronco.Com relação às alterações posturais, evidenciaram-se melhoras em curto prazo em relação a alguns aspectos, tais como a protrusão do ombro e cabeça, a anteversão pélvica e a protrusão abdominal. Contudo, é indicada a continuidade da intervenção, a fim de obter maiores ganhos posturais. Pontes $^{24}$ vai ao encontro desta afirmativa, ao comentar que o método Pilates, aplicado em curto prazo, ocasiona melhora postural em alguns aspectos; porém, para obter ganhos mais significativos na reeducação postural, é necessário um maior número de sessões em um período maior. $\mathrm{O}$ fato, porém, de se obter melhora com menor número de sessões pode ser relevante em casos em que não seja possível, por algum motivo, realizar um tratamento mais prolongado.

Considerando que a escoliose promove desequilíbrios de força e comprimento musculares no tronco, apresentando a musculatura do lado côncavo de maneira retraída e músculos mais alongados no lado convexo da curvatura, o que caracteriza um problema de assimetria muscular, é necessário que a intervenção fisioterapêutica inclua a estabilização e a simetria muscular como foco de tratamento. ${ }^{25} \mathrm{Um}$ estudo investigou a ativação muscular paravertebral em uma menina de nove anos de idade, com diagnóstico clínico de escoliose idiopática. Como resultado, os autores identificaram uma assimetria da ativação dessa musculatura, e levantam a ideia que este desequilíbrio muscular contribua para a progressão da curvatura escoliótica. ${ }^{26}$ Corroborando com essa hipótese, foi encontrada uma redução da força dos músculos extensores de tronco na avaliação da participante do presente estudo, fato este que pode ter contribuído para a formação da curvatura escoliótica.

Assim, um programa de estabilização central, incluindo exercícios de fortalecimento dos músculos paravertebrais, deve ser indicado como meio de reabilitação em alterações posturais importantes, ${ }^{7}$ a exemplo da escoliose. Adicionalmente, Kolyniak et al. ${ }^{27}$ mostraram que o método Pilates foi eficaz para o aumento da função dos músculos extensores de tronco, além do discreto aumento para o trabalho total dos músculos flexores de tronco em jovens saudáveis, efeitos estes percebidos também na participante do presente estudo.

Estudos demonstram que o método Pilates promove a estabilização lombo-pélvica e abdominal, além da flexibilidade. ${ }^{10,28} \mathrm{O}$ treinamento da estabilização central, ou do core, que representa a base do método, consiste no recrutamento dos músculos multifídios e transverso do abdômen em atividades funcionais, pois a contração desses músculos aumenta a rigidez do tronco e a pressão intra-abdominal, com carga mínima para a coluna lombar. ${ }^{29}$

Alguns dados sugerem que os músculos multifídios apresentam maior ativação muscular com os exercícios do método Pilates, do que quando comparados com outros métodos, como Série de Williams e Spine Stabilization. ${ }^{30}$ Logo, os exercícios do método Pilates, a exemplo do exercício do cisne na bola, utilizado no atual estudo, denotam uma ação importante sobre a ativação desse grupo muscular estabilizador de tronco. O grau de fortalecimento proporcionado pelo Pilates contribui para um melhor alinhamento postural, devido a uma melhor relação entre músculos agonistas e antagonistas, relacionados ao equilíbrio postural e articular, otimizando os quadros de escoliose. ${ }^{31}$ Assim, provavelmente devido ao fato de que os exercícios do método Pilates recrutam a musculatura estabilizadora, especialmente os multifídios, foi evidenciado, na participante do estudo, um aumento de força dos músculos extensores de tronco, ao final da intervenção.

Existem evidências de que o método Pilates promove um incremento significativo sobre a flexibilidade de indivíduos com alterações posturais. ${ }^{32} \mathrm{Da}$ mesma forma, o estudo de Araújo et al. ${ }^{33}$ evidenciou que a prática do método Pilates foi eficaz na redução da dor, aumento da flexibilidade e melhora de alterações posturais, em estudantes do gênero feminino, portadoras de escoliose não estrutural. Apesar de não ter sido mensurado o índice de dor, a participante do presente estudo obteve resultados importantes após a intervenção, especialmente relacionados com a flexibilidade, onde foram evidenciadas melhoras nos dois testes utilizados (testes de sentar alcançar e da distância dedo-chão) e nas alterações posturais (melhora na protrusão do ombro e cabeça, anteversão pélvica e protrusão abdominal).

Como limitações do estudo pode-se citar o curto período de tempo no qual foi realizada a intervenção. Esse curto período justifica-se pelo fato de que requisição médica solicitava 10 sessões fisioterapêuticas, sendo este um número de sessões frequentemente solicitado 
pelos médicos através do Sistema Único de Saúde. Outra limitação está relacionada ao fato de não ter sido confirmada a redução da escoliose pelo padrão ouro, ou seja, a avaliação radiológica pelo ângulo de Cobb, antes e após a intervenção.

Apesar dessas limitações, este estudo demonstrou que após a intervenção baseada no método Pilates ocorreu uma evidente melhora no quadro clínico dessa adolescente com escoliose idiopática. Apesar do curto período de tempo, houve melhora postural no alinhamento de cabeça, ombros, escápulas, ângulo de Talles, tronco, abdômen e pelve; e ainda melhora na flexibilidade e no fortalecimento dos músculos flexores e extensores de tronco.

A divulgação destes resultados pode fornecer subsídios para novos estudos e contribuir para o planejamento de estratégias de intervenção, no sentido de prevenir a instalação prematura e/ou tardia de alterações posturais em crianças e adolescentes, além de promover a conscientização de hábitos saudáveis.

\section{REFERÊNCIAS}

1. Asher MA, Burton DC. Adolescent idiopathic scoliosis: natural history and long term treatment effects. Scoliosis. 2006;1(2):1-10.

2. Dohnert MB, Tomasi E. Validade da fotogrametria computadorizada na detecção de escoliose idiopática adolescente. Rev Bras Fisioter. 2008;4(12):290-7.

3. Rocha EST, Pedreira ACS. Problemas ortopédicos comuns na adolescência. J Pediatr. 2001;77(2):225-233.

4. Malina RM, Bouchard C. Atividade física do atleta jovem: do crescimento à maturação. São Paulo: Roca; 2002.

5. Weinstein SL, Dolan LA, Cheng JC, Danielsson A, Morcuende JA. Adolescent idiopathic scoliosis. Lancet. 2008;371(9623):1527-37.

6. Yaman O, Dalbayrak S. Idiopathic Scoliosis. Turk Neurosurg. 2014; 24(5): 646-57.

7. O'Sullivan PB. Lumbar segmental "instability": clinical presentation and specific stabilizing exercise management. Man Therapy. 2000;5(1):2-12.

8. Toledo PCV, Mello DB, Araújo ME, Daoud R, Dantas EHM. Efeitos da Reeducação Postural Global em escolares com escoliose. Fisioter Pesq. 2011;18(4):329-34.

9. Cardoso LR, Gonçalves C, Bonvicine C, Barboza MAI. Análise clínica e radiográfica pré e pós-tratamento conservador na escoliose idiopática do adolescente: estudo de caso. Consc Saúde. 2011;10(1):166-72.

10. Kloubec J. Pilates: how does it work and who needs it? Muscles Ligaments Tendons J. 2011;1(2):61-6.

11. Segal NA, Hein J, Basford JR. The Effects of Pilates Training on Flexibility and Body Composition: An Observational Study. Arch Phys Med Rehabil. 2004;85(12):1977-81.

12. Braz RG, Goes FPDC, Carvalho GA. Confiabilidade e Validade de Medidas Angulares por meio do Software para Avaliação Postural. Fisioter Mov. 2008;21(3):117-26.

13. Martinelli AR, Purga MO, Mantovani AM, Camargo MR, Rosell AA, Fregonesi CEPT, Freitas Junior IF. Análise do alinhamento dos membros inferiores em crianças com excesso de peso. Rev Bras Cineantropom Des Hum. 2011;13(2):124-30.

14. American College of Sports Medicine. Diretrizes do ACSM para os testes de esforço e sua prescrição. $6^{\underline{a}}$ ed. Rio de Janeiro: Guanabara Koogan; 2007.

15. Brasil. Ministério do Esporte. Projeto Esporte Brasil (PROESP-BR). Indicadores de saúde e fatores de prestação esportiva em crianças e jovens. [Internet]. Desenvolvido pelo Setor de Pedagogia do Esporte do CENESP-UFRGS. [updated 2012; cited 2013 Sept 30] Available from: http://www.esporte.gov.br/arquivos/snear/talentoEsportivo/proespBr.pdf

16. Carregaro RL, Silva LCCB, Gil Coury HJC. Comparação entre dois testes clínicos para avaliar a flexibilidade dos músculos posteriores da coxa. Rev Bras Fisioter. 2007;11(2):139-45.

17. Daniels L, Williams M, Worthingham C. Le testing. 2a ed. Paris: Maloine; 1958.

18. Araújo MEA, Silva EB, Vieira PC, Cader AS, Mello DB, Dantas EHM. Redução da dor crônica associada à escoliose não estrutural, em universitárias submetidas ao método Pilates. Motriz. 2010;16(4):958-66.

19. Dias TB, Mejia DPM. Incidência de escoliose em crianças de 12 a 15 anos em idade escolar. Pós-graduação em Fisioterapia em Reabilitação na Ortopedia e Traumatologia com ênfase em Terapia Manual - Faculdade Ávila [updated 2013 Jan; cited 2013 Sep 27] Available from: http://www.portalbiocursos.com.br/artigos/ortopedia/08.pdf

20. Zurita OF, Moreno LC, Ruiz RL, Martínez MA, Zurita OA, Castro SAM. Screening of scoliosis in a school population of 8 to 12 years in the province of Granada (Spain). An Pediatr. 2008;69(4):342-50.

21. Minghelli B. Rastreio escolar: a importância na detecção precoce de posturas escolióticas em adolescentes das escolas de Silves, Algarve. Rev Port Saúde Pub. 2008;26(2):61-8.

22. Pereira LM, de Barros PCC, de Oliveira MND, Barbosa AR. Escoliose: triagem em escolares de 10 a 15 anos. Rev Saúde Com. 2005;1(2):134-43.

23. Iunes DH, Cecílio MBB, Dozza MA, Almeida PR. Análise quantitativa do tratamento da escoliose idiopática com o método Klapp por meio de biofotogrametria computadorizada. Braz J Phys Ther. 2010;14(2):133-40. 
24. Pontes S. Efeitos do Método Pilates no alongamento da cadeia posterior. Rev Nova Fisio \& Terap. 2008;60:23-4.

25. Bassani E, Candotti CT, Pasini M, Melo M, La Torre M. Avaliação da ativação neuromuscular em indivíduos com escoliose através da eletromiografia de superfície. Braz J Phys Ther. 2008;12(1):13-9.

26. Tosato JP, Caria PHF. Assessment of the muscle activity on scoliosis. Rev Bras Cresc Desenv Hum 2009;19(1):98-102.

27. Kolyniak IEGG, Cavalcanti SMB, Aoki MS. Avaliação isocinética da musculatura envolvida na flexão e extensão do tronco: efeito do método Pilates. Rev Bras Med Esporte. 2004;10(6):487-90.

28. Phrompaet S, Paungmali A, Pirunsan U, Sitilertpisan P. Effects of Pilates Training on Lumbo-Pelvic Stability and Flexibility. Asian J Sports Med. 2011;2(1):16-22.

29. Wilson JD, Dougherty CP, Ireland ML, Davis IM. Core stability and relationship to lower extremity function and injury. J Am Acad Orthop Surg. 2005;13(5):316-25.

30. Silva MAC, Dias JM, Silva MF, Mazuquin BF, Abrão T, Cardoso JR. Análise comparativa da atividade elétrica do músculo multífido durante exercícios do Pilates, série de Williams e Spine Stabilization. Fisioter Mov. 2013;26(1):87-94.

31. Ferreira CB, Aidar FJ, Novaes GS, Vianna JM, Carneiro AL, Menezes LS. O método Pilates sobre a resistência muscular localizada em mulheres adultas. Motricidade. 2007;3(4):76-81.

32. Sinzato CM, Taciro C, Pio CA, Toledo AM, Cardoso R, Carregaro RL. Efeitos de 20 sessões do método Pilates no alinhamento postural e flexibilidade de mulheres jovens: estudo piloto. Fisioter Pesqui. 2013;20(2):143-50.

33. Araújo MEA, Silva EB, Mello DB, Cader SA, Salgado ASI, Dantas EH. The effectiveness of the Pilates method: reducing the degree of non-structural scoliosis, and improving flexibility and pain in female college students. J Bodyw Mov Ther. 2012;16(2):191-8. 